\title{
FRECUENCIA DE RIESGO DEL SÍNDROME DE APNEA OBSTRUCTIVA DEL SUEÑO EN LA POBLACIÓN QUE ASISTE A TRES (3) IPS ODONTOLÓGICAS DE BUCARAMANGA
}

\author{
${ }^{1}$ Jorge Alberto Anaya Florez, ${ }^{1}$ Nilton José Joaquín García Parada, ${ }^{2}$ Liliana Margarita Otero Mendoza, ${ }^{3}$ Diana Marina Camargo Lemos \\ ${ }^{1}$ Odontólogo, Residente tercer año, posgrado de Ortodoncia, U. Santo Tomás. \\ ${ }^{2}$ Ortodoncista, M.Sc Microbiologia y Genetica, U. Javeriana. ${ }^{3}$ M Sc Epidemiología, Profesora auxiliar \\ Escuela de Fisioterapia, U. Industrial de Santander
}

Autor responsable de correspondencia: Dr. Jorge Alberto Anaya F.

Correo electrónico: betaya12@hotmail.com

\section{RESUMEN}

Objetivo: Determinar la frecuencia de riesgo del Síndrome de Apnea Obstructiva del Sueño (OSA), en la consulta de odontología-ortodoncia en tres (3) Instituciones Prestadoras de Salud (IPS) de la ciudad de Bucaramanga

Materiales y métodos: Se realizó un estudio de corte transversal, se seleccionaron 503 individuos de ambos géneros con edades entre 19 -72 años, a quienes se les aplicó el test de Epworth y el test de Berlin, además se recolectó información relacionada con antecedentes médicos y variables sociodemográficas generales. Se determinó la frecuencia de alto riesgo de OSA y sus intervalos de confianza del 95\%. La evaluación de posibles asociaciones entre antecendentes médicos y variables sociodemográficas con el alto riesgo de OSA se determinó aplicado modelos de regresión binomial simple, considerando un $\alpha=0.05$.

Resultados: Se encontró un 7.4\%, IC 95\% (5.2 - 10) de alto riesgo de apnea con el test de Berlin y de 16.5\%, IC 95\% (13.2 19.8) con el test de Epworth. La mayoría de antecedentes clínicos se asociaron en forma significativa con el alto riesgo de apnea $(\mathrm{p}<0.05)$, situación contraria para género y edad.

Conclusiones: Determinar en forma precoz el riesgo de apnea, favorecerá una intervención interdisciplinaria que contribuira mejorar la calidad de vida del paciente. [Anaya JA, García NJJ, Otero LM, Camargo DM. Frecuencia del riesgo de apnea obstructiva del sueño en la población que asiste a tres (3) IPS odontológicas de Bucaramanga. Ustasalud Odontología 2003; 2: 90 - 96]

PALABRAS Cl.AVE: Apnea obstructiva del sueño, ronquido, hipersomnolencia diurna, test de Berlín, test de Epworth.

\section{Frequency of the obstructive sleep apnea syndrome in three dentistry and orthodontic clinics from} Bucaramanga

\begin{abstract}
Purpose: To determine the frequency of the obstructive sleep apnea syndrome in three dentistry and orthodontic clinics in the city of Bucaramanga.

Material and methods: A cross sectional study was done. Five hundred and three patients were analyzed in demographic variables, clinical signs and symptoms. The Berlin and Epworth tests were applied to determine the high risk for sleep apnea in the population. The analysis included binomial regression models to evaluate association between high risk of sleep apnea and demographic and clinical characteristics.

Results: The frequency of sleep apnea was 7.4\%, IC 95\% (5.2 - 10) (37 individuals) by Berlin test and 16.5\%, IC 95\% (13.2 19.8) by Epworth test. Significative associations were found between clinical signs and symptoms and sleep apnea $(\mathrm{p}<0.05)$. However, gender and age were not associated.

Conclusions: An earlier diagnosis of sleep apnea contributes to the patient quality of life and better interventions by the health team.
\end{abstract}

KEY WORDS: Obstructive sleep apnea, apnea, snoring, Berlin test, Epworth test. 


\section{INTRODUCCIÓN}

Se ha observado un creciente interés en la comunidad medica por estudiar los desórdenes o patologías del sueño, ya que se han convertido en un problema de salud pública de gran trascendencia, por la morbimortalidad asociada. ${ }^{1}$

La Apnea Obstructiva del Sueño (AOS) es una patología del sueño, que se produce por la obstrucción intermitente y repetida del espacio faríngeo superior 0 vía aérea superior durante el sueño, lo que origina una interrupción completa (apnea) o parcial (hipoapnea) del flujo aéreo, su interés radica en los problemas que genera, como la morbimortalidad relacionada con accidentes de tránsito y laborales, cardiopatías isquémicas y cor pulmonar crónico. Se caracteriza principalmente por un evento nocturno que presenta episodios recurrentes de obstrucción de las vías aéreas superiores, el colapso faríngeo y el cese del flujo aéreo transitorio (arousal o bífido). ${ }^{1,2}$

Presenta una prevalencia alta, superior al $5 \%$ en hombres adultos de 40 - 65 años, aunque hay otros estudios que reportan prevalencia un 2 - 4\% de la población adulta entre 20 y 60 años. Siendo el ronquido habitual nocturno, uno de los síntomas más característicos, no todos los individuos que roncan sufren de apnea obstructiva del sueño; sin embargo, más del $25 \%$ de las personas por encima de los 65 años presentan más de cinco (5) apneas del sueño por hora. ${ }^{2-5}$

A nivel local hay un solo estudio que determinó una prevalencia de alto riesgo de apnea, de $6.6 \%$ con un intervalo de confianza del 95\%, (5.1\% - 8.3\%), en consulta odontológica, mediante el test de Epworth y algunos signos y síntomas clínicos. ${ }^{5}$

El objetivo de esta investigación fue determinar la frecuencia de riesgo del Síndrome de Apnea Obstructiva del Sueño (SAOS), en la consulta de odontología - ortodoncia en tres (3) Instituciones Prestadoras de Salud (IPS) de la ciudad de Bucaramanga. Además, describir las características socio - demográficas de la población según los niveles de riesgo y los estados de somnolencia, evaluando algunos antecedentes médicos (signos y síntomas) relacionados con el alto riesgo de apnea.

\section{MATERIALES Y MÉTODOS}

Se realizó un estudio de corte transversal, seleccionando una muestra de 503 individuos de ambos géneros mayores de 18 años, que asistieron a la consulta de odontología y ortodoncia en las clínicas odontológicas de la Universidad Santo Tomás, IPS de la Universidad y Salud TOTAL, entre septiembre de 2002 hasta febrero de 2003.

Se analizaron variables sociodemográficas como edad, género, índice de masa corporal I.M.C y perímetro de cuello; antecedentes médicos: Ronquido, diabetes, problemas cardiacos, somnolencia diurna, consumo de medicamentos, nicturia, impotencia sexual, cefalea matutina, mal sabor de boca y garganta seca en las mañanas, problemas de concentración, alto riesgo de apnea, establecido con los test de Epworth ${ }^{7}$ y de Berlín. ${ }^{8}$

La escala de somnolencia de Epworth ${ }^{7}$ reune 8 preguntas en una escala de categorías ordenada de 0 - 3 , donde 0 es nunca se ha dormido y 3 es elevada posibilidad de dormirse. Al totalizar los puntajes de Epworth se obtiene un rango posible entre 0 y 24 . Posteriormente, los puntajes fueron dicotomizados en los individuos que tuvieron un puntaje mayor de 11, definido como alto riesgo de presentar apnea del sueño.

El cuestionario de Berlín consta de una pregunta introductoria y cuatro preguntas relacionadas con el ronquido; tres preguntas sobre la somnolencia diurna, una de hipersomnolencia cuando se conduce un vehículo automotriz. Adicionalmente, indaga sobre hipertensión arterial, edad, peso, altura, sexo, circunferencia del cuello y grupo étnico. ${ }^{8}$

Siguiendo las recomendaciones de Netzer se definieron tres categorías de síntomas: ${ }^{8}$

Categoría 1: Definida como síntomas persistentes, mayores 3 - 4 veces por semana en dos (2) o más preguntas relacionadas con ronquido.

Categoría 2: Definida como persistencia mayor de 3 - 4 veces por semana de somnolencia diurna, dormirse conduciendo vehículo o ambos.

Categoría 3: Definida como hipertensión arterial e índice de masa corporal mayor de 30.

Los puntajes de Berlín determinaron que los pacientes que califiquen por lo menos a dos categorías de síntomas pueden ser considerados de alto riesgo de presentar apnea 
obstructiva del sueño. Aquéllos que negaron tener síntomas persistentes o quienes calificaron sólo para una categoría de síntomas, se registró en el grupo de bajo riesgo de presentar apnea obstructiva del sueño. ${ }^{8}$

\section{Análisis}

Después de recoger la información se realizó una descripción general de las variables realizando medidas de tendencia central y dispersión o tablas frecuencia, según la naturaleza y distribución de cada variable. ${ }^{9}$

Posteriormente se aplicó un análisis bivariado, con el fin de evaluar posibles asociaciones entre algunos factores sociodemográficos con el nivel de riesgo de apnea, según los formularios de Berlín y de Epworh, para tal fin se aplicaron inicialmente pruebas como $\mathrm{X}^{2}$ para las variables categóricas y t de Student o ANOVA para promedios con dos o más grupos de comparación respectivamente. ${ }^{9}$ Posteriormente se aplicaron regresiones binomiales simples, con el objetivo de estimar la magnitud y dirección de la asociación mediante razones de prevalencia, entre los factores de exposición y el alto riesgo de apnea. ${ }^{10,11} \mathrm{La}$ Base datos se elaboró en Excel ${ }^{12}$ y el análisis en STATA 6.0. ${ }^{13}$

Considerando la Resolución 008430 de 1993 del Ministerio de Salud, esta investigación se incluyó en la categoría de riesgo mínimo, ya que sólo se aplicaron procedimientos de medición simples y se recolectó la información mediante entrevista directa con el paciente. Se respetaron además los principios éticos de veracidad, confidencialidad y autonomía durante todo el trabajo.

\section{RESULTADOS}

Se evaluaron un total de 503 individuos mayores de 18 años, entre $19-72$ años con un promedio de $36.7 \pm 10$ años, Los datos relacionados con la variables de antecedentes médicos se presentan en la Figura 1, de la cual sobresalen los porcentajes del ronquido (44.7\%), somnolencia diurna (35.7\%), consumo de medicamentos antihipertensivos como el captopril, enalapril, bloqueadores de la secreción gástrica (ranitidina, famotidina, nizatidina), nicturia (31.2\%), alteraciones del sabor y las mucosas secas en las mañanas (51.4\%), y dificultad para concentrarse (48.3\%).

Al evaluar el IMC y el perímetro de cuello de la población estudiada, el IMC presentó un rango de 13.1 - $44.12 \mathrm{kgr} / \mathrm{m}^{2}$ con un promedio de $24.44 \mathrm{kgr} / \mathrm{m}^{2} \pm 3.94 \mathrm{kgr} / \mathrm{m}^{2}$. Al categorizar el IMC en mayor de 30, se encontró un porcentaje de $9 \%$ en el total de la población. No se encontraron asociaciones entre IMC mayor de 30 con edad o género $(\mathrm{p}>0.05)$.

El perímetro de cuello de los individuos estuvo entre $28 \mathrm{y}$ $54 \mathrm{~cm}$ con un promedio de $37.1 \mathrm{~cm} \pm 4.73 \mathrm{~cm}$. Para el análisis bivariado se trabajó como variable dicotómica y se establecieron dos categorías en un punto de corte $=$ $36 \mathrm{~cm}$, con base en la mediana de la distribución. Se encontró significativamente mayor para el género masculino.

Los resultados de la escala de somnolencia de Epworth se muestran en la Tabla 1 . Se encontraron puntajes entre 0 y 23, con un promedio de $6.6 \pm 4.7$. Al establecer la categoría de alto riesgo con puntajes superiores a 11, se encontró una frecuencia de 16.5\%, IC 95\% (13.2 - 19.8).

Considerando las recomendaciones de Netzer ${ }^{8}$ de los resultados generales del Cuestionario de Berlín se muestran en la Tabla 5, de la cual sobresalen el porcentaje de ronquido (43.3\%) y las variables relacionadas con la frecuencia e intensidad del ronquido. En cuanto a la frecuencia de cansancio diurno, las frecuencias fueron inferiores al $12 \%$, en general.

El análisis por categorías de riesgo según el test de Berlín detectó en la categoría 1: 122 individuos (24.25\%), categoría 2: 36 individuos (7.16\%), categoría 3: 1 individuo $(0.2 \%)$.

Con base en estas categorías se estableció que un alto riesgo de presentar apnea obstructiva del sueño está representado para aquellos indicadores en categorías mayor 0 igual a dos (2), por lo tanto el porcentaje de alto riesgo de apnea obstructiva del sueño en la muestra fue de 7.4\%, (37 individuos) IC 95\% (5.2 y 10)con el test de Berlin.

La evaluación de posibles asociaciones entre las variables de estudio con el alto riesgo de apnea según el test de Berlin se muestra en la Tabla 2. Se encontraron asociadas en forma significativa la presencia de enfermedades cardiovasculares RR 3.05, IC95\% (1.88 - 4.95), nicturia, RR 20.5 , IC95\% (1.39 - 3.02) y cefalea matutina RR 1.91, IC 95\% $(1.29-2.82)$ entre otras.

\section{2}


Tabla 1. Resultados Escala de Somnolencia de Epworth (n: 503).

\section{SITUACIÓN}

\section{CATEGORIAS}

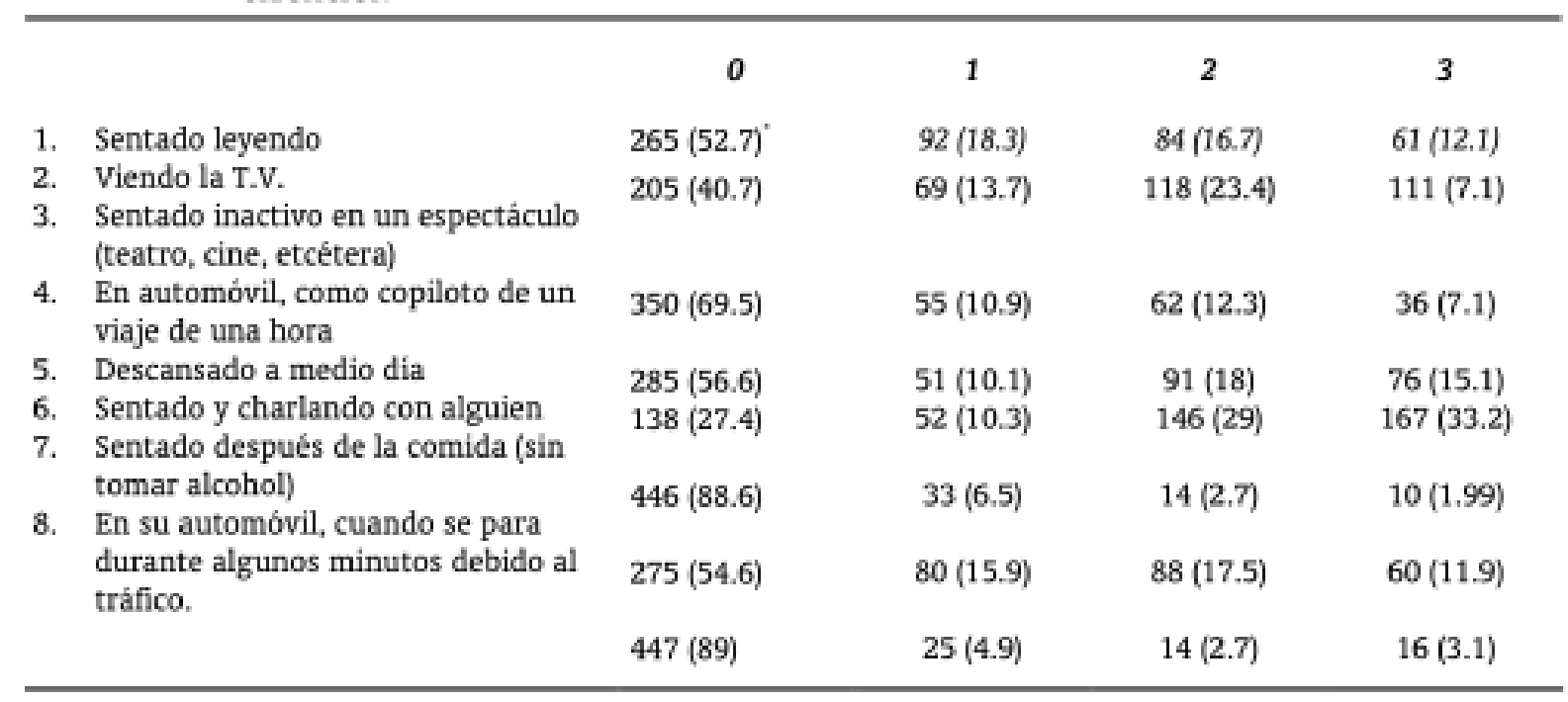

* No (\%)

Tabla 2. Análisis bivariado entre las variables sociodemográficas y algunos antecedentes médicos con el alto riesgo de apnea establecido con el test de Epworth.

\begin{tabular}{|c|c|c|c|c|}
\hline Variable & $\begin{array}{c}\text { Alto Riesgo } \\
\quad=11 \\
\mathrm{~N}: 83\end{array}$ & $\begin{array}{c}\text { Bajo Riesgo } \\
<11 \\
\mathrm{~N}: 420\end{array}$ & $\begin{array}{c}\text { RR } \\
\text { [ IC 95\% ] }\end{array}$ & $\mathrm{p}$ \\
\hline Género masculino & 43 & 185 & $\begin{array}{c}1.29 \\
{[0.87-1.92]} \\
1.07\end{array}$ & 0.19 \\
\hline Edad>35 años & 45 & 219 & $\begin{array}{c}{[0.72-1.59]} \\
1.17\end{array}$ & 0.73 \\
\hline Diabetes & 8 & 384 & $\begin{array}{c}{[0.61-2.26]} \\
3.05\end{array}$ & 0.64 \\
\hline E.C.V. & 11 & 13 & $\begin{array}{c}{[1.88-4.95]} \\
0.98\end{array}$ & 0.01 \\
\hline Consumo de medicamentos & 28 & 144 & $\begin{array}{c}{[0.65-1.49]} \\
2.05\end{array}$ & 0.92 \\
\hline Nicturia & 40 & 117 & $\begin{array}{c}{[1.39-3.02]} \\
1.88\end{array}$ & $<0.0001$ \\
\hline Impotencia sexual & 25 & 69 & $\begin{array}{c}{[1.24-2.83]} \\
1.91\end{array}$ & 0.003 \\
\hline $\begin{array}{l}\text { Cefalea matutina } \\
\text { Alteraciones de sabor de boca y }\end{array}$ & 35 & 104 & $\begin{array}{l}{[1.29-2.82]} \\
2.19\end{array}$ & 0.001 \\
\hline mucosas secas & 58 & 201 & $\begin{array}{c}{[1.41-3.88]} \\
2.63\end{array}$ & $<0.0001$ \\
\hline Dificultad para concentrarse & 59 & 184 & $\begin{array}{c}{[1.69-4.09]} \\
1.73\end{array}$ & $<0.0001$ \\
\hline Perimetro de cuello > $35 \mathrm{~mm}$ & 58 & 230 & {$[1.12-2.67]$} & 0.013 \\
\hline
\end{tabular}


Tabla 3. Análisis bivariado entre las variables sociodemográficas y algunos antecedentes médicos con el alto riesgo de apnea establecido con el test de Berlin.

\begin{tabular}{|c|c|c|c|c|}
\hline Variable & $\begin{array}{l}\text { Alto Riesgo } \\
\quad=2 \\
\mathrm{~N}: 37\end{array}$ & $\begin{array}{c}\text { Bajo Riesgo } \\
<2 \\
\text { N:466 }\end{array}$ & $\begin{array}{c}\text { RR } \\
\text { [ IC 95\% ] }\end{array}$ & p \\
\hline Género masculino & 21 & 207 & $\begin{array}{c}1.60 \\
{[0.85-2.96]} \\
1.89\end{array}$ & 0.15 \\
\hline Edad $>35$ años & 25 & 239 & $\begin{array}{l}{[0.97-3.67]} \\
2.12\end{array}$ & 0.06 \\
\hline Diabetes & 24 & 240 & $\begin{array}{c}{[0.94-4.80]} \\
3.86\end{array}$ & 0.07 \\
\hline $\begin{array}{l}\text { E.C.V. } \\
\text { Consumo de medicamentos }\end{array}$ & 6 & 18 & $\begin{array}{l}{[1.78-8.40]} \\
2.82\end{array}$ & 0.01 \\
\hline & 22 & 150 & $\begin{array}{c}{[1.50-5.30]} \\
3.23\end{array}$ & 0.01 \\
\hline Nicturia & 22 & 135 & $\begin{array}{c}{[1.72-6.10]} \\
2.65\end{array}$ & $<0.0001$ \\
\hline Impotencia sexual & 14 & 80 & $\begin{array}{c}{[1.42-4.95]} \\
3.44\end{array}$ & 0.002 \\
\hline $\begin{array}{l}\text { Cefalea matutina } \\
\text { Alteraciones de }\end{array}$ & 21 & 118 & {$[1.84-6.39]$} & $<0.0001$ \\
\hline $\begin{array}{l}\text { Alteraciones de sabor de boca y } \\
\text { mucosas secas } \\
\text { Dificultad para concentrarse }\end{array}$ & 29 & 230 & $\begin{array}{c}3.41 \\
{[1.59-7.32]} \\
2.89\end{array}$ & 0.002 \\
\hline \multirow{2}{*}{ Perimetro de cuello $>35 \mathrm{~mm}$} & 27 & 216 & $\begin{array}{c}{[1.43-5.84]} \\
3.85\end{array}$ & 0.03 \\
\hline & 31 & 257 & {$[1.64-9.10]$} & 0.002 \\
\hline
\end{tabular}

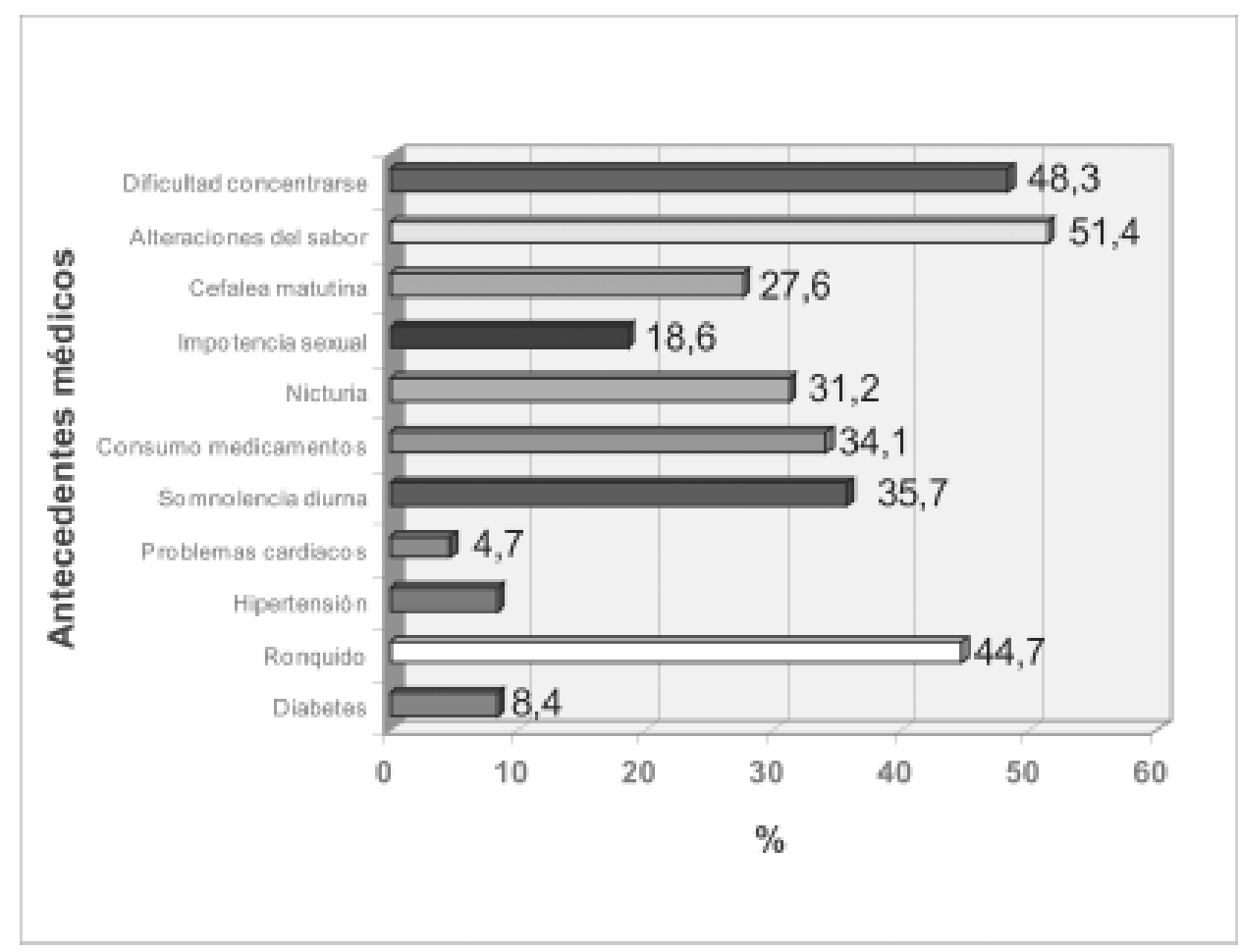

Figura 1. Distribución de los antecedentes médicos en la población de estudio ( $n=503)$. 
La evaluación de las posibles asociaciones entre las variables socio-demográficas y los antecedentes médicos con el alto riesgo de presentar apnea obstructiva del sueño, establecido con el cuestionario de Berlín, se presentan en la Tabla 3. Se detectó como factor de riesgo ser mayor de 35 años, RR 1.89, IC 95\% (0.97 - 3.67). Es importante señalar que el 45.9\% (17/37) individuos de alto riesgo son mayores de 40 años.

La mayoría de los antecedentes médicos estuvieron asociados en forma estadísticamente significativa con el alto riesgo de apnea obstructiva del sueño a excepción de género (Tabla 3). Los pacientes obesos IMC $>30$ de alto riesgo son $13 / 37$ con un porcentaje $35.1 \%$. Los pacientes hipertensos de alto riesgo son 11/37 (29.7\%).

Los datos muestran que el promedio de perímetro de cuello es mayor para el grupo de alto riesgo de presentar apnea obstructiva del sueño con un promedio de $40.8 \mathrm{~cm}$, comparado con los de bajo riesgo con un promedio de $36.91 \mathrm{~cm}$. Al establecer la categoría de riesgo en este variable, se dicotomizó por la mediana de la distribución, es decir perímetro de cuello $=36 \mathrm{~cm}$. Por lo tanto el RR calculado correspondió a 3.85, IC 95\% (1.64 - 9.10) (Tabla 3).

\section{DISCUSIÓN}

La importancia de establecer la prevalencia de AOS en la población e identificar posibles indicadores de riesgo asociados, tanto en la consulta odontológica y de ortodoncia, como en la médica, radica en el reconocimiento precoz de los signos de la enfermedad, para ser atendido por un equipo interdisciplinario para definir la terapéutica adecuada. $^{14}$

Varios estudios epidemiológicos han establecido una prevalencia de $S A O S$ en población general y mayor de 30 años, entre 2 y 4\%, 5,14 Sin embargo se ha sugerido que probablemente esta cifra sea mayor del 5\%, en especial en la población adulta mayor. ${ }^{14}$ Nuestros hallazgos en la consulta odontológica y de ortodoncia, muestran una frecuencia mayor, pues con base en el test de Berlín se estimó una cifra de $7.4 \%$ con un IC 95\% (5.2 10\%).

Es posible que la diferencia se deba a la selección de la muestra, pues mientras la mayoría de estudios tienen como base la población general, en éste estudio se trabajó con una muestra pre-seleccionada, proveniente de las consultas de ortodoncia y odontología, de tres IPS de
Bucaramanga. Este aspecto igualmente se constituye en una limitante del estudio, por lo cual no se pudo establecer la prevalencia, sino la frecuencia relativa estimada en porcentaje.

A nivel local, tan sólo se dispone de un estudio preliminar, realizado en la consulta odontológica de Bucaramanga. ${ }^{6}$ Este trabajo estableció una prevalencia de alto riesgo de apnea del 6.6\%, IC 95\% (5.1 - 8.3), definiendo un posible caso de apnea con un test de Epworth $=11$, diagnóstico de hipertensión arterial y ronquido nocturno. Al compararlos con los hallazgos del trabajo actual, no se perciben diferencias significativas.

El análisis de la frecuencia de alto riesgo de apnea por edad, mostró una media de edad de 39 años para el grupo de alto riesgo y de 35 para el de bajo riesgo con una significancia marginal $(\mathrm{p}=0.06)$. Por lo anterior, la edad se categorizó en dos grupos, estableciendo como punto de corte la media en 35 años, para el análisis bivariado. El porcentaje de alto riesgo de apnea en mayores de 35 años fue de $9.5 \%$, IC95\% (6.2 - 13.7) y de 5\%, IC 95\% (2.6 - 8.6) para los menores de 35 años.

Se estableció una razón hombre mujer de 1.59 con unas frecuencias e intervalos de confianza de $9.2 \%(5.7$ - 13.7) y 5.8 (3.4 - 9.3), respectivamente, inferior a la estimada en las publicaciones previas, pues presentan una razón de 2:1, respectivamente. ${ }^{14}$ La explicación de este fenómeno se da probablemente por la predisposición del hombre a colapsar más la faringe por diferentes causas en relación a la mujer : por características estructurales (anatomía local) y características funcionales (laxitud de los tejidos para ambos sexos), con mecánicas para la vía aérea más favorables en las mujeres, por lo cual no colapsan, a excepción de las mujeres posmenopáusicas, quienes por su componente hormonal disminuido, generan un aumento en la frecuencia de AOS.

El rol del ortodoncista en el diagnóstico precoz del SAOS, ya ha sido planteado pues puede intervenir terapéuticamente al paciente con aparatología, aumentando la columna de aire que debe pasar por el espacio faríngeo superior colapsado, además, si el ortodoncista corrige la apnea obstructiva del sueño también contribuye a disminuir la manifestación posterior de hipertensión arterial, somnolencia diurna, y otras alteraciones relacionadas con el $S A O S .^{14-16}$ 


\section{BIBLIOGRAFÍA}

1. Strollo P, Rogers R. Obstructive sleep apnea. New England J Med 1996; 334: 99 - 104.

2. Rowley J. Obstructive sleep apnea. Med J 2000; 3.

3. Guilleminault C, Partinen M, Quera - Salva M, Hayes B, Dement W, Nino-Murcia G. Determinants of Determinants of daytime sleepiness in obstructive sleep apnea. 1988; 94: 7-32.

4. Alvarez - Sala W, Calle R, Fernández A. Apnea Obstructiva del Sueño. En: Información Terapéutica del Sistema Nacional de Salud. 1999; 23 (5). URL disponible en: http://www.msc.es/farmacia/infmedic.

5. Young T, Palta M, Dempsey J, Skatrud J, Weber S, Badr S. The occurrence of sleep disordered breathing among middle aged adults. New England Journal of Medicine. 1993; 328: 1230 - 1235.

6. Carrillo A, García NC. Romero ME. Prevalencia del Síndrome de Apnea Obstructiva del Sueño en la consulta odontológica de Bucaramanga y su área metropolitana. [Tesis de Grado]. Bucaramanga: Universidad Santo Tomás; 2001.

7. Johns MW. A new method for measuring daytime sleepiness: The Epworth Sleepiness Scale. Sleep. 1991; 14: 540 - 545.

8. Netzer N, Stoohs R. Using the Berlin questionnaire to identify patients at risk for the sleep apnea syndrome. Annals of Internal Medicine. 1999; 131: 485 - 491.

9. Pagano M, Gauvreau K. Principles of biostatistics. Duxbury Press, Belmont Ca, 1993.

10. Wacholder S. Binomial Regresión in GLIM: Estimating risk and risk differences. American Journal of Epidemiology. 1986; 123: 174 - 184.

11. Zochetti C, Consonni D, Vertáis PA. Relationship between prevalence rate ratios and odds ratios in cross - sectional studies. International Journal of Epidemiology 1997; 26: 220 - 223.

12. Microsoft Excel, 5.0, Microsoft Corporation, 1997.

13. StataCorp. 1999. Stata Statistical Software: Release 6.0. College Station, TX: Stata Corporation.

14. Guilleminault C, Partinen M. Obstructive sleep apnea syndrome. Clinical research and treatment. New Raven Press; 1990.

15. Dancey D, Hanly P, Soong $\mathrm{CH}$. Impact of menopause on the prevalence and severity of sleep apnea. 2001; 120: 151 - 155.

16. Malhotra A, Huang Y, Pillar G, White D. The male predisposition to pharyngeal collapse, importance of airway length. American Journal of Respiratory and Critical Care Medicine 2002; 166: 1388 - 1395. 